\title{
DNAJB6 Promotes Ferroptosis in Esophageal Squamous Cell Carcinoma
}

\author{
Bin Jiang ${ }^{1} \cdot$ YongQiang Zhao ${ }^{2} \cdot$ Mo Shi $^{1} \cdot$ Liang Song $^{1} \cdot$ Qiang Wang ${ }^{1} \cdot$ QiMing Qin $^{1} \cdot$ XueMin Song $^{1} \cdot$ Shuo Wu $^{1}$. \\ Zhen Fang ${ }^{3} \cdot$ XiangYan Liu' ${ }^{1}$
}

Received: 6 July 2019 / Accepted: 31 October 2019 / Published online: 8 November 2019

(c) The Author(s) 2019

\begin{abstract}
Background DnaJ/Hsp40 homolog, subfamily B, member 6 (DNAJB6) is significantly down-regulated in esophageal squamous cell carcinoma (ESCC), while its complicated molecular mechanisms are still unknown.

Aims To investigate the relationship between DNAJB6 and ESCC.

Methods The expression of DNAJB6 was detected in ESCC patient by Western blot and immunohistochemistry. To overexpress DNAJB6a by lentivirus infection, colony-forming, CCK-8, transwell, mouse xenograft assays were utilized to verify the proliferous, invasive, and migratory role of DNAJB6a in ESCC cells. The MDA and GSH assays determine whether DNAJB6a participates in cell redox reaction. The variation of AKT and GPX4 was detected by Western blot.

Results The correlation between DNAJB6 level and lymph node metastasis in ESCC patient was negative. Overexpressing DNAJB6a shows tumor-suppressive effects in vitro and in vivo. In addition, DNAJB6a overexpression was accompanied together with a remarkable reduction in the protein levels of GPX4 and phosphorylated AKT (p-AKT).
\end{abstract}

Conclusion DNAJB6 plays an important anti-oncogenic role in ESCC evolvement via ferroptosis.

Keywords DNAJB6 $\cdot$ ESCC $\cdot$ AKT $\cdot$ GPX4 $\cdot$ Ferroptosis

\section{Introduction}

Esophageal carcinoma is a quite common digestive tumor in the world. It has two main pathological types: esophageal adenocarcinoma (EAC) and esophageal squamous cell carcinoma (ESCC). EAC is common in western countries, while ESCC is the dominant pathological type in Asia including
China [1]. ESCC progresses rapidly and metastasizes early. Hence, it is of great importance to research the molecular mechanism of ESCC for treatment and prognosis.

DnaJ/Hsp40 homolog, subfamily B, member 6 (DNAJB6) is also known as mammalian relative of DnaJ (MRJ). Human DNAJB6 has two spliced isoforms: isoform a $(2.5 \mathrm{~kb}$, NM_058246) and isoform b (1.6 kb, NM_005494). The

\author{
XiangYan Liu \\ liuxiangyan1@163.com \\ Bin Jiang \\ jiang9958888@163.com \\ YongQiang Zhao \\ 342302825@qq.com \\ Mo Shi \\ legendsm01@163.com \\ Liang Song \\ mylikest07@163.com \\ Qiang Wang \\ wq19790620@126.com \\ QiMing Qin \\ qqmhzjqqm@163.com
}

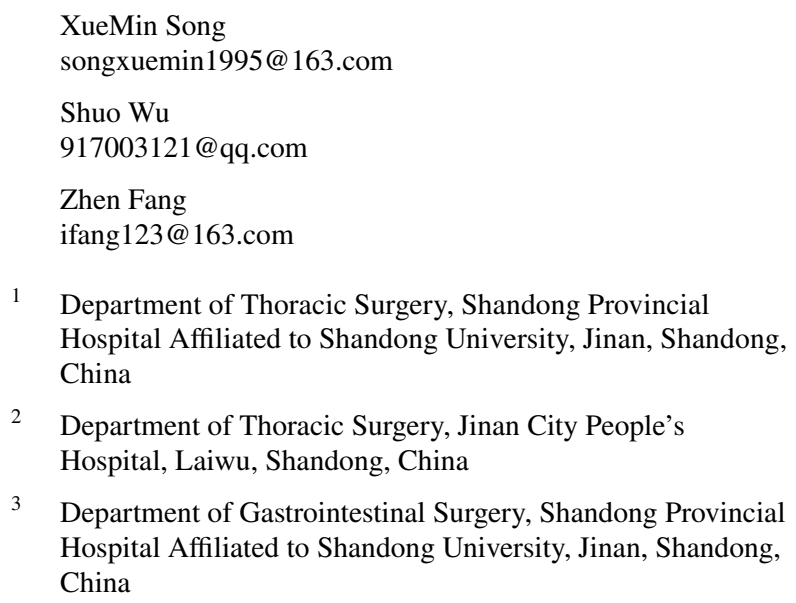

1 Department of Thoracic Surgery, Shandong Provincial Hospital Affiliated to Shandong University, Jinan, Shandong, China

2 Department of Thoracic Surgery, Jinan City People's Hospital, Laiwu, Shandong, China

3 Department of Gastrointestinal Surgery, Shandong Provincial Hospital Affiliated to Shandong University, Jinan, Shandong, China 
isoform a of DNAJB6 comprises 326 amino acids, while the isoform b includes 242 amino acids. A lot of nervous system disease, for example Parkinson's disease, Huntington disease, and Parkinsonian syndrome, show protein aggregation of DNAJB6 [2]. Nevertheless, the function of DNAJB6 in carcinoma has not been clear.

Recent research showed there is an iron-dependent form of cell death termed ferroptosis in some cancer cells [3]. Unlike cell necrosis and apoptosis, ferroptosis is caused by lipid peroxides when ferrous iron reacts with it. Inhibiting defensive protein called glutathione peroxidase 4 (GPX4, an antioxidant enzyme) may selectively activate ferroptosis [4]. GPX4 plays an important role in holding back ferroptosis through decreasing phospholipid hydroperoxide and hence inhibiting lipid peroxidation [4-6]. GPX4 lowers the lipid peroxides so that the iron no longer works [7]. In addition, GPX4 is necessary for tumor recurrence [8]. When ferroptosis occurs, mitochondrial morphology and function change as follows: Decreased mitochondria are accompanied by an increase in the density of the bilayer membrane and loss of its structural integrity, moreover the decreased mitochondrial transmembrane potential and reduced oxidative phosphorylation $[9,10]$. Some scholars had hypothesized that ferroptosis would be a treatment method of malignancies; however, the extent to which this method could work was unclear [11].

\section{Patient and Method}

\section{Immunohistochemistry (IHC)}

DNAJB6 antibody (1:250 dilution) was used as the primary antibody. The intensity of staining of tissue was assessed as 0 (no staining) to 3 (strongest intensity of staining). The scores $\leq 2$ were defined as low expression, whereas scores $>2$ were defined as high expression. The Olympus light microscope was used to magnify and for observation.

\section{Western Blot}

Protein was extracted both from tissues and cell lines. An equal amount of protein sample $(20 \mu \mathrm{g})$ was resolved on SDS-PAGE and immunoblotted for levels of various proteins. Commensurable loading was confirmed by $\beta$-actin and tubulin reference. Experiments were repeated three times independently.

\section{Patients}

We performed immunohistochemical and Western blot analyses of ESCC tissues and adjacent histologically normal tissues from 64 patients, who underwent esophagectomy without preoperative radiotherapy and chemotherapy at Shandong Provincial Hospital from September 2017 to December 2018. The samples were surplus specimens after diagnosing. All samples were stored at $-80{ }^{\circ} \mathrm{C}$ until analysis. The immunohistochemical results were evaluated separately by two independent pathologists. All patients participating in the study signed informed consents. And the study protocol was approved by the Institutional Ethics Committee of Shandong Provincial Hospital Affiliated to Shandong University.

\section{Cell Lines and Culture}

Human ESCC cell lines (TE-1, Eca9706, Eca109, KYSE150, and KYSE450) were obtained from the cell repository of Chinese Academy of Sciences. The cells were cultured in RPMI 1640 with $10 \%$ fetal bovine serum (FBS), $1 \%$ penicillin/streptomycin, and $1 \% \mathrm{~L}$-glutamine. Cell culture flasks were maintained at $37{ }^{\circ} \mathrm{C}$ in incubator with $5 \% \mathrm{CO}_{2}$.

\section{Lentivirus Infection}

To overexpress the DNAJB6a, we got the lentivirus (NM_058246) and the control lentivirus from GeneChem Company, Shanghai, China. The experimental methods followed manufacturer's manual. After infection, the cells were screened by puromycin to obtain stable transfection cell lines.

\section{Reverse Transcription Quantitative Polymerase Chain Reaction (RT-qPCR)}

Whole-cell RNA was extracted via the RNAiso Plus (TaKaRa, Japan). The cDNA samples were amplified using the SYBR Green qPCR assay kit (Takara) and the LightCycler480 PCR system. The following primers were used for RT-qPCR: DNAJB6a primers (GeneChem): forward 5'-ATC GGAAACTGGCACTGAAG-3' and reverse 5'-TCTGGG TTACGGAATGTGAA-3'; $\beta$-actin primers (Takara): forward 5'-AGAGCCTCGCCTTTGCCGATCC-3 'and reverse 5'-ATACACCCGCTGCTCCGGGTC-3'. Three independent experiments were performed to analyze the relative DNAJB6 expression level.

\section{Colony-Forming Assay}

Cells were seeded in 6-well plates and medium was changed every 3 days. The plates were incubated at $37{ }^{\circ} \mathrm{C}$ in $5 \% \mathrm{CO}_{2}$. The plates were fixed with formaldehyde and stained with $0.5 \%$ crystal violet. The results were calculated by the average number of colonies in plates. The assay was performed in triplicates. 


\section{CCK-8 Assay}

The cells proliferation was detected by cell counting kit- 8 (CCK-8, Dojindo, Japan). Cells were seeded in 96-well plates within $100 \mu \mathrm{l}$ culture medium and incubated with CCK8 reagent $\left(10 \mu \mathrm{l} /\right.$ well) for $2 \mathrm{~h}$ at $37{ }^{\circ} \mathrm{C}$ incubator. The absorbance of each well was measured by microplate reader at an excitation of $450 \mathrm{~nm}$.

\section{Transwell Assay}

To detect the capacity of cell migration and invasion, transwell assay was carried out by using a 24 -well cell culture insert with $8-\mathrm{mm}$ pore (3422, Corning, USA). The uncoated inserts were used in the migration assay, while Matrigel (1:6 dilution; 354234, Corning, USA) was precoated to the upper surface of inserts in the invasion assay. Cells were pre-treated by culturing in serum-free medium overnight. In the lower chamber of the 24-well plates, RPMI 1640 culture media containing 20\% FBS was used as a chemotactic attractant. Single-cell suspensions were loaded in the upper space of inserts. Cells from the upper surface of the inserts were removed by cotton swab. The underneath cells were fixed with formaldehyde and stained with $0.5 \%$ crystal violet. The membranes were sealed by neutral gum and observed by Olympus microscope.

\section{Mouse Xenograft Assay}

Female BALB/c athymic nude mice (4 weeks of age) were obtained from the HFK Bioscience Co, Beijing. To generate murine subcutaneous tumors, $2 \times 10^{6}$ Eca109 cells and KYSE 150 cells in $100 \mu \mathrm{l}$ PBS were injected subcutaneously on the left of the nude mice's dorsal midline. The xenografts were measured every 4 days, and volumes were calculated using the formula: (length $\times$ width $\left.^{2}\right) / 2$. Three weeks after injection, the xenografts were removed. After being removed, the xenografted tumor was detected by IHC as previously mentioned.

\section{Lipid Peroxidation Assay}

The malondialdehyde (MDA) was detected by Lipid Peroxidation MDA Assay Kit (S0131, Beyotime, Beijing) according to the manufacturer's direction. The absorbance was measured at $532 \mathrm{~nm}$ after $2 \mathrm{~min}$ and compared to a standard curve of MDA.

\section{Determination of Glutathione (GSH) Levels}

The levels of GSH in the cells were determined according to the method based on the Glutathione Detection Reagent (T10095, Invitrogen, USA). Fluorescence intensity was detected by FCM.

\section{Transmission Electron Microscope}

The mitochondrial morphology of different cell lines was shot by transmission electron microscope (HITACHI HT7700) after fixation, embedding, and staining.

\section{Statistical Analysis}

The statistical data were presented in the form of mean \pm standard. $P$ values were calculated by the analysis of variance and Chi-squared test. It is considered to be statistically different if $P$ value $<0.05$. All statistical calculations and graph were performed using GraphPad Prism 7 statistical software.

\section{Result}

\section{There Was a Negative Correlation Between DNAJB6 Level and Lymph Node Metastasis in ESCC Patient}

We detected the DNAJB6 level by Western blot and IHC. Western blot analysis showed that DNAJB6a expression was lower in ESCC tissues compared with the matched normal esophageal tissues (Fig. 1A). IHC results indicated that DNAJB6 expresses high in normal tissues. Meanwhile, high or low expression was detected in different ESCC tissues (Fig. 1B). Combined with the postoperative pathological report, we found that compared to patients with high DNAJB6 levels, ESCC patients who have low DNAJB6 levels in tumor tissues were more prone to have lymph node metastasis (Table 1). However, there is no relation between DNAJB6 and other factors, such as age, gender, grade, and T stage.

\section{DNAJB6a Expression Is Up-Regulated by Lentivirus Infection}

Cell culture, protein extraction, and Western blot analysis were as previously mentioned. The result (Fig. 2A) shows that Eca 109 and KYSE 150 have a lower expression level in DNAJB6a. Thus, we choose Eca 109 and KYSE 150 to carry out subsequent experiments. The transfection efficiency was verified by Western blot (Fig. 2B-C). RT-qPCR reveals that DNAJB6a was increased compared with the bland and control group (Fig. 2D). 
Fig. 1 DNAJB6a expression was lower in ESCC tissues compared with the matched normal esophageal tissues. Note a The DNAJB6 expression of 8 representative tissue sample pairs detected by Western blot. N, normal tissue; $\mathrm{T}$, tumor tissue. b The expression of DNAJB6 in ESCC tissues and normal esophagus tissues was detected by $\mathrm{IHC}(\times 200)$

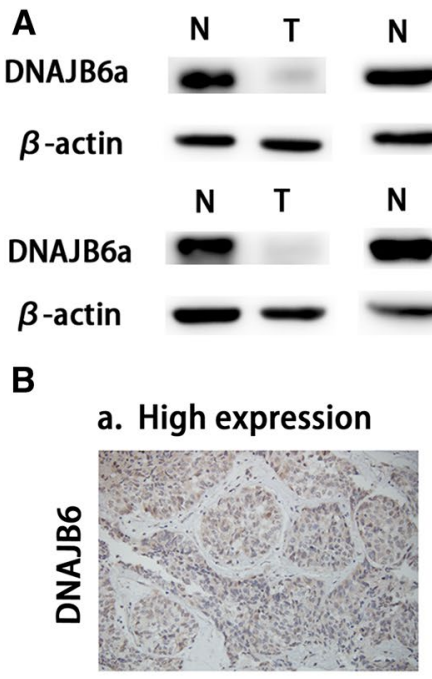

Tumor tissue b. Low expression

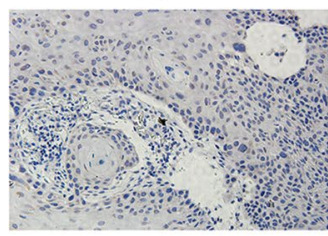

Tumor tissue

\section{c. High expression}

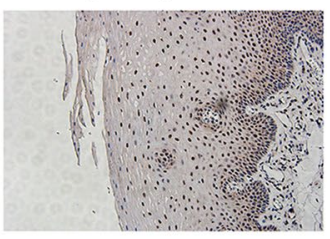

Normal tissue
Table 1 Clinical characteristics and its relationship with DNAJB6 expression

\begin{tabular}{|c|c|c|c|c|}
\hline pathological feature & Total & $\begin{array}{l}\text { High nuclear } \\
\text { DNAJB6 }\end{array}$ & $\begin{array}{l}\text { Low nuclear } \\
\text { DNAJB6 }\end{array}$ & $P$ value \\
\hline AGE & & & & 0.902 \\
\hline$\geq 60$ & 42 & 14 & 28 & \\
\hline$<60$ & 22 & 7 & 15 & \\
\hline Gender & & & & 0.728 \\
\hline Male & 47 & 16 & 31 & \\
\hline Female & 17 & 5 & 12 & \\
\hline Grade & & & & 0.796 \\
\hline Well/moderate & 35 & 11 & 24 & \\
\hline Poor & 29 & 10 & 19 & \\
\hline T stage & & & & 0.141 \\
\hline $\mathrm{T} 1-\mathrm{T} 2$ & 44 & 17 & 27 & \\
\hline T3-T4 & 20 & 4 & 16 & \\
\hline $\begin{array}{l}\text { Lymph node metas- } \\
\text { tasis }\end{array}$ & & & & $\mathbf{0 . 0 2 3}^{*}$ \\
\hline Positive & 40 & 9 & 31 & \\
\hline Negative & 24 & 12 & 12 & \\
\hline M stage & & & & - \\
\hline M0 & 64 & 21 & 43 & \\
\hline M1 & 0 & 0 & 0 & \\
\hline
\end{tabular}

Bold value indicates that the result has statistical significance

\# Calculated by Chi-squared test * $p<0.05$

\section{Overexpressing DNAJB6a Shows Tumor-Suppressive Effects In Vitro}

The colony-forming assay shows that the colony count in overexpressing DNAJB6a group is markedly decreased compared with blank and control groups (Fig. 3A-B), which indicates that DNAJB6a overexpression suppresses the proliferation of ESCC cells. The same result can be obtained from CCK8 assay (Fig. 3C). Meanwhile, the transwell assay shows that compared with the blank and control groups, there are less cells passing through the membrane in DNAJB6a overexpression group (Fig. 3D-G). These results indicate that the overexpressing DNAJB6a weakens the migratory and invasive capacity of ESCC cells.

\section{Overexpressing DNAJB6a Weakens Tumorigenesis in Mouse Model}

Compared to the blank and control groups, the average xenografts volume of overexpressing DNAJB6a was significantly decreased. The Eca 109 and KYSE 150 cell lines show greater tumor-suppressive effects by DNAJB6a overexpression (Fig. 4).

\section{DNAJB6a Participates in Cell Redox Reaction}

The measurement of MDA and GSH in ESCC cells indicates that DNAJB6a overexpression enhanced lipid peroxidation (Fig. 5A) and reduced the GSH (Fig. 5B-C). Hence, we concluded that overexpressing DNAJB6a promotes oxidation reaction of ESCC cells. In addition, DNAJB6a overexpression cells contained smaller mitochondria and higher membrane density, mitochondrial structures lose their integrity, and edema occurs in the mitochondrial matrix (Fig. 5D). 
Fig. 2 The endogenous and exogenous expression of DNAJB6a in cell lines. Note a The expression of DNAJB6a in different cell lines. b Western blot showing the exogenous up-regulation of DNAJB6a. c Further verification of the transfection efficiency by anti-flag antibody. d Relative DNAJB6a mRNA level after transfection. The results are expressed as the mean \pm SD. $* * * p<0.001$

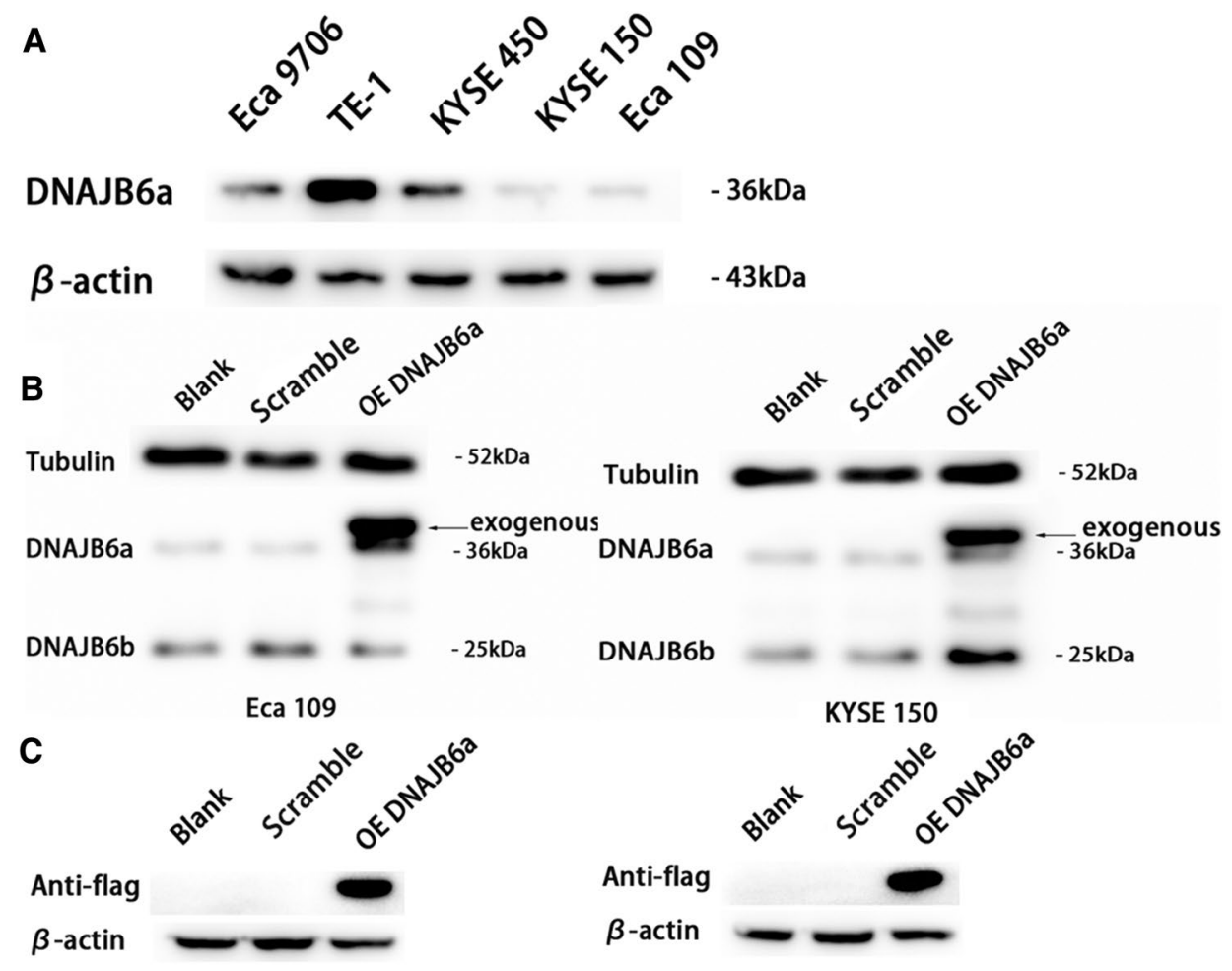

Eca 109

KYSE 150

D

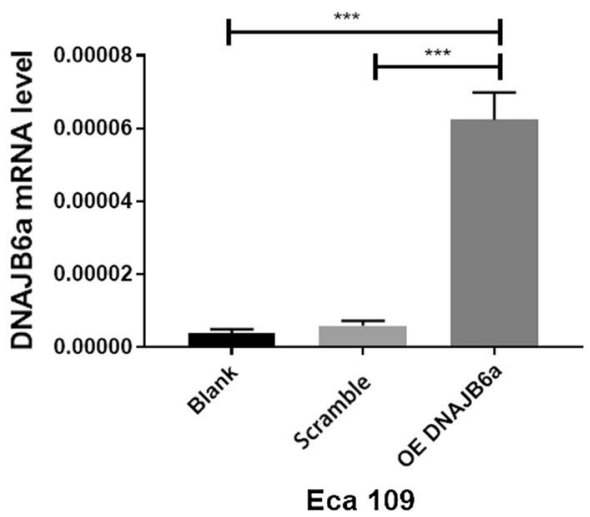

\section{Overexpressing DNAJB6a Gene Was Accompanied by a Conspicuous Reduction in the Protein Levels of GPX4 and p-AKT}

We detected the decreasing phosphorylation of AKT and GPX4 in Eca109 and KYSE150 by Western blot after treatment with overexpressing DNAJB6a (Figure 6).

\section{Discussion}

ESCC is a frequent malignant tumor of gastrointestinal cancer in the world. Its poor prognosis is mainly caused by lymphatic metastasis at the early stage and local recurrence [12]. Therefore, it is very crucial to find the potential therapeutic target by studying the mechanism of tumor metastasis.

The DNAJB6 gene encodes two splice variants: isoforms $a$ and $b$. The preceding research found that DNAJB6a localizes to the nucleus due to the functional NLS located at the C-terminal. Meanwhile, the proliferation inhibition regulated by DNAJB6a is AKT-dependent [13]. What is more, DNAJB6a was detected to regulate the $\mathrm{Wnt} / \beta$-catenin signaling pathway $[14,15]$. Based on these, our research mainly explores the role of the isoform a in ESCC.

The present research makes a further verification via different methods that DNAJB6 plays a tumor-suppressive role 
A

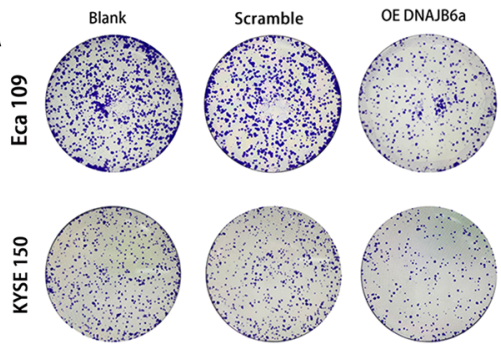

C

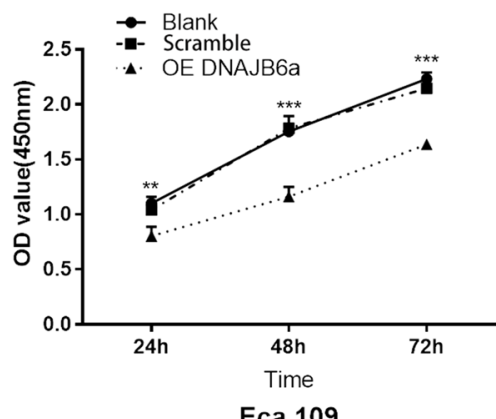

Eca 109

D
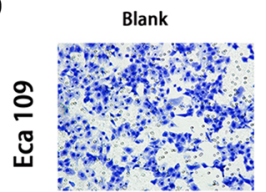

Scramble
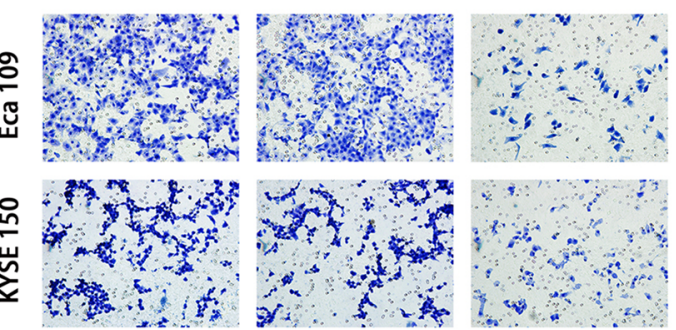

$\mathbf{F}$

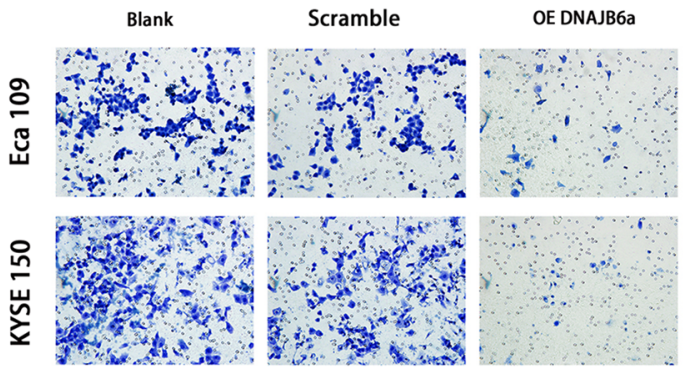

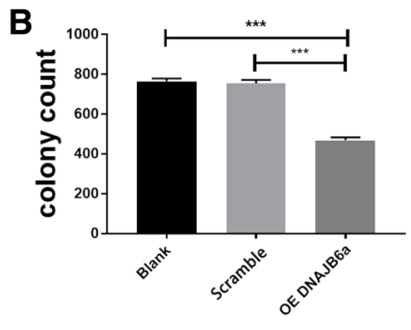

Eca 109

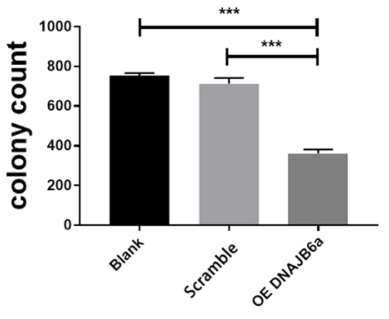

KYSE 150

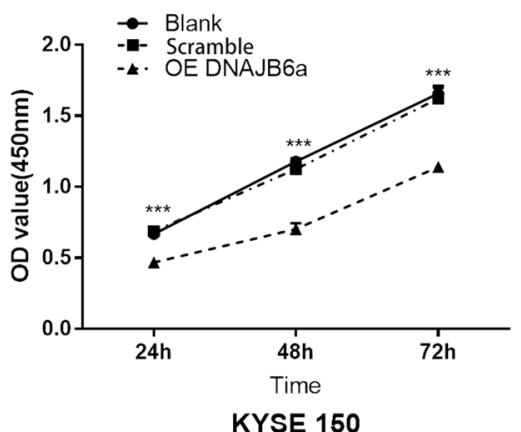

KYSE 150

E

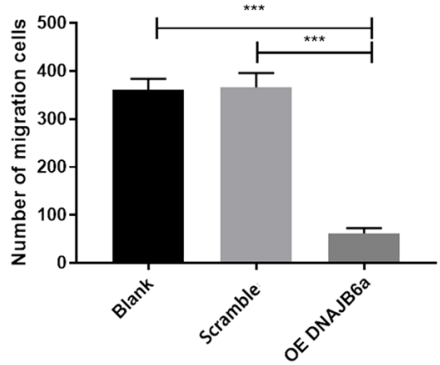

Eca 109

G

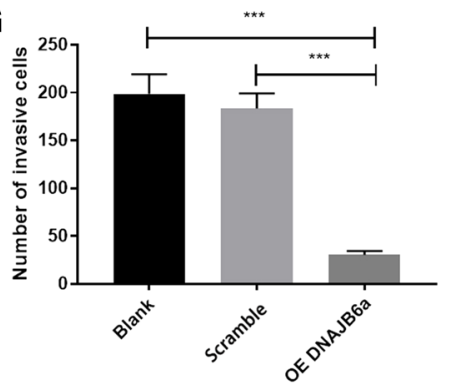

Eca 109

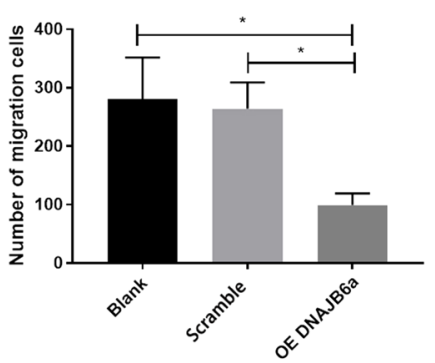

KYSE 150

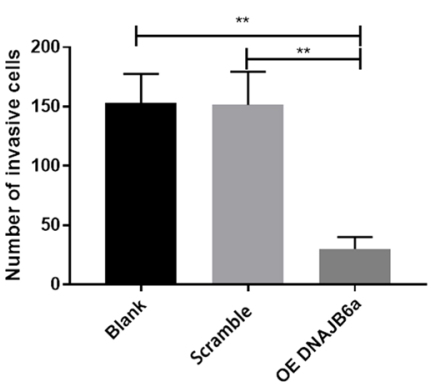

KYSE 150
Fig. 3 Overexpressing DNAJB6a shows tumor-suppressive effects in vitro. Note a The Eca 109 and KYSE 150 cells were seeded in 6-well plate at a density of 1000 cells/well; after 10 days, the cells were disposed as what mentioned before. b The colony count is expressed in the form of mean \pm SD of three replicates. c Cells were seeded on 96-well plates with $100 \mu$ of culture medium at a density of 5000 cells/well. OD values were measured after 24,48 and $72 \mathrm{~h}$. The results are expressed as the mean \pm SD of three replicates. $d$ The cells were sowed in the 24-well transwell plates at the concentration of $5 \times 10^{5}$ cells $/ \mathrm{ml}$ in $0.2 \mathrm{ml}$ serum-free medium in the upper com- partment. And $500 \mu \mathrm{l}$ of culture medium with $20 \%$ FBS was added to the lower compartment. After $48 \mathrm{~h}$, cells were processed as previously mentioned. Micrographs were taken by optical microscopy $(\times 200)$. e The cell that passed through the membrane was counted in three random fields. $\mathbf{f}$ The cells were seeded into the upper compartment pre-coated with $50 \mu \mathrm{l}$ Matrigel. Other conditions as mentioned above. $g$ The cell that passed through the membrane were counted in three random fields. The data were expressed as mean \pm SD of three independent repeated experiments. $* p<0.05, * * p<0.01$, $* * * p<0.001$ 
A

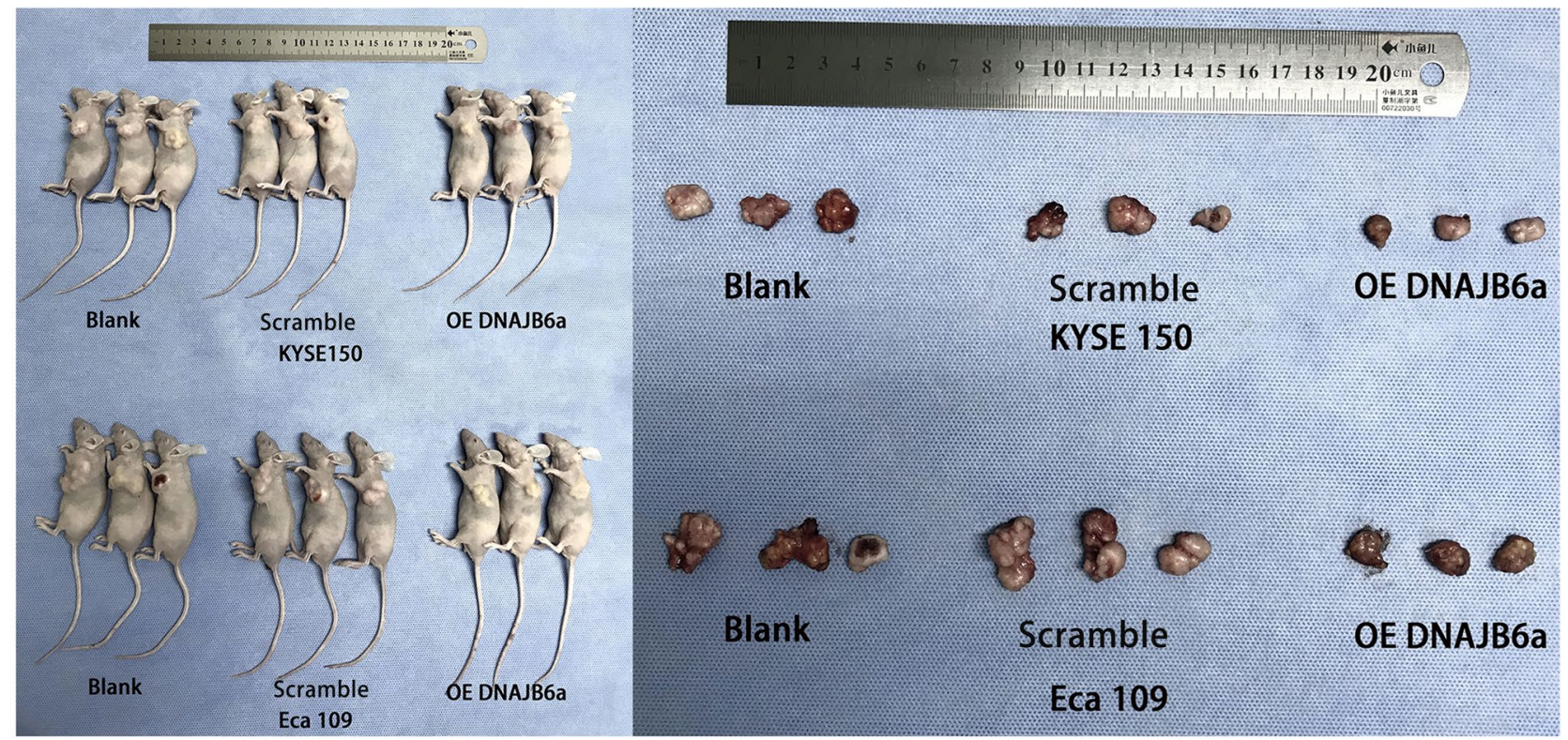

B
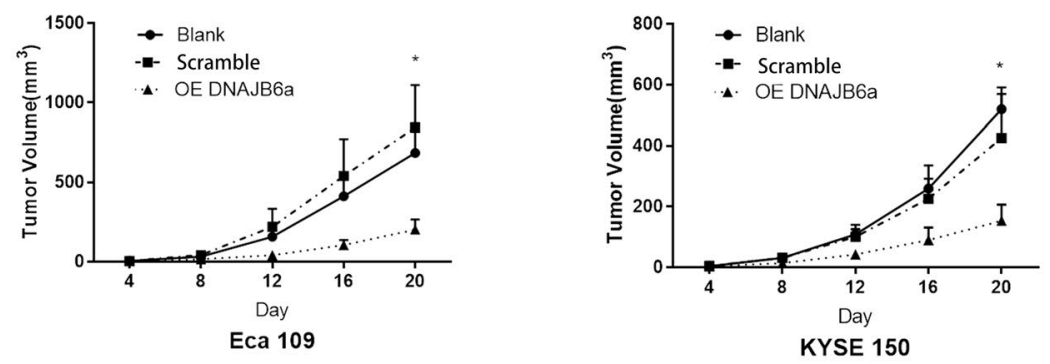

C
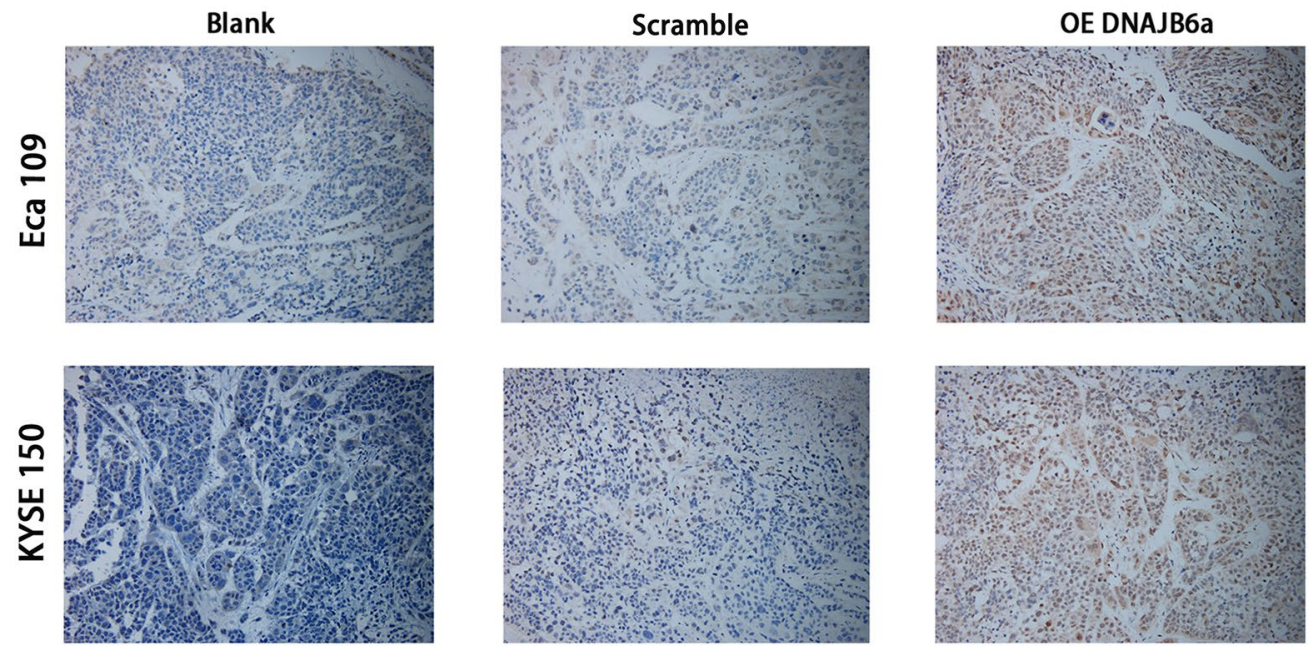

Fig. 4 Overexpressing DNAJB6a inhibits xenograft growth in vivo. Note a Nude mice were executed after 20 days since injected subcutaneously. b The results are expressed as tumor volume (mean \pm SD).

in ESCC. Furthermore, we reveal its tumor-suppressive role from different aspects.

First of all, according to the results of IHC and Western blot, we found that DNAJB6 expression was down-regulated in ESCC tissues compared with the matched normal
${ }^{*} p<0.05$. c IHC showed that the xenografts in blank and negative control show low expression, and in the positive control show high expression

esophageal tissues. Combined with the postoperative pathological report, we found that compared to patients with high DNAJB6 levels, ESCC patients who have low DNAJB6 levels in tumor tissues were more prone to lymph node metastasis. These results imply that DNAJB6 dysregulation may 

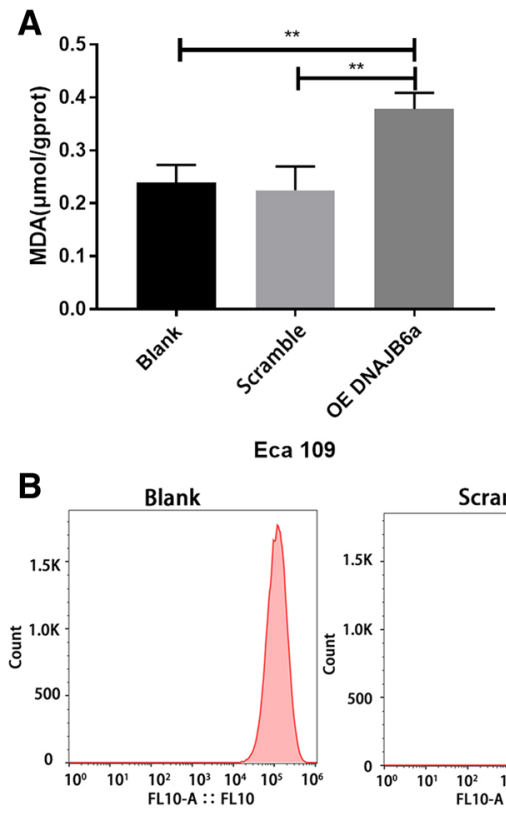

ca 109

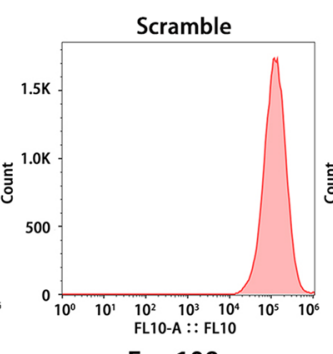

Eca 109
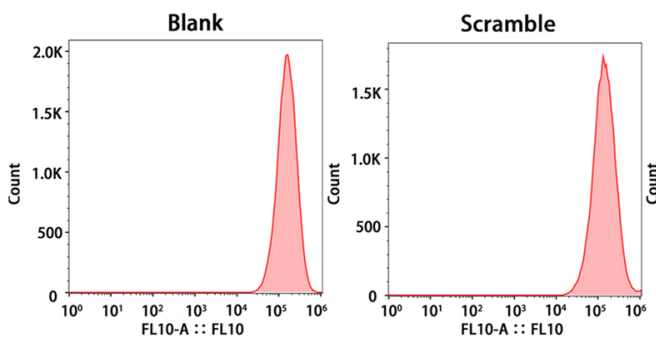

KYSE 150

D

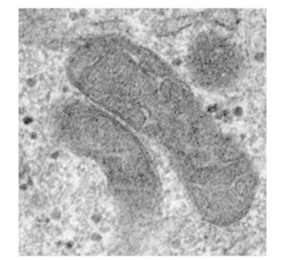

Blank

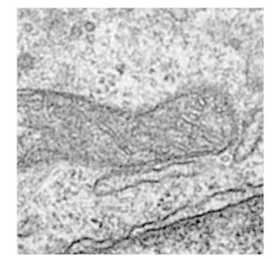

Scramble

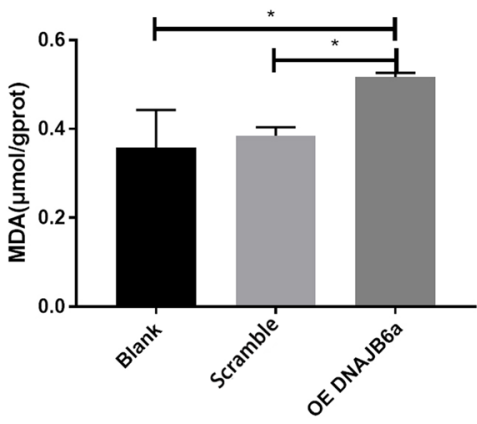

KYSE 150
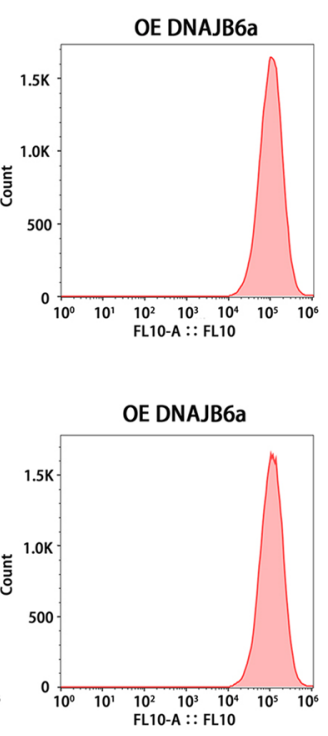

C
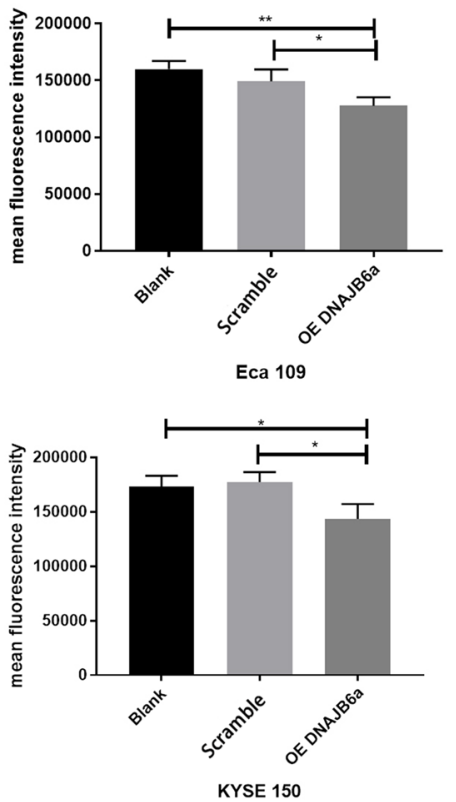

Eca 109

Fig. 5 DNAJB6a is related to cell redox reaction. Note a The relative amount of MDA proofread by protein. $\mathbf{b}$ The fluorescence intensity of GSH detected by FCM. c Quantitative fluorescence intensity data are

play a significant role in the progress of the ESCC. Hence, we supposed DNAJB6 acts as an anti-oncogene in ESCC.

To verify the hypothesis, we carried out further experiments in vitro. Western blot shows that DNAJB6a expression is lower in Eca 109 and KYSE 150 cell lines compared to others. Based on this result, we choose Eca 109 and KYSE 150 cell lines to build DNAJB6a overexpression model by lentivirus. The transfection efficiency was verified by RTqPCR and Western blot. We observed that compared to the

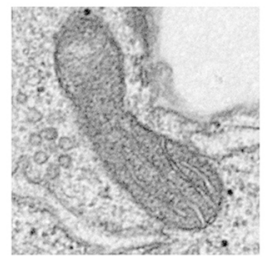

Blank

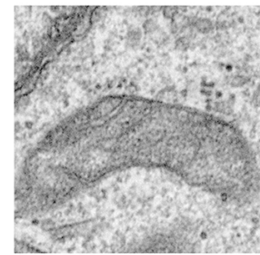

Scramble KYESE 150

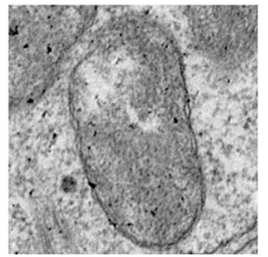

OE DNAJB6a calculated by FlowJo and presented as mean \pm SD. d Transmission electron microscopic images of Eca 109 and KYSE 150 cells, $6 \times 10^{3}$ magnification. $* p<0.05, * * p<0.01$

blank and control groups, overexpressing DNAJB6a had noteworthy impediment on cell proliferation, migration, and invasion, together with the decreased $\mathrm{p}$-AKT expression. In vivo, the result showed that DNAJB6a overexpression decreased the rate of tumor growth. In consequence, we can confirm that DNAJB6 acts as an anti-oncogene in ESCC.

Ferroptosis is a regulatable way of cell death; lipid peroxidation plays an important role in mediating it $[11,16]$. GPX4 is a kind of the GSH peroxidase [17]. And previous study 


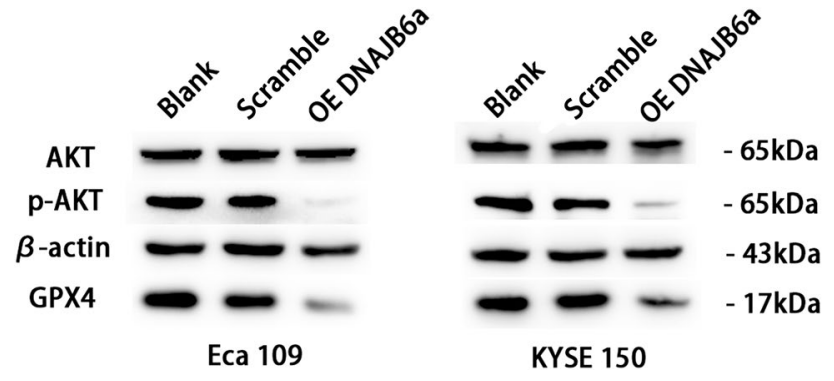

Fig. 6 Overexpressing DNAJB6a gene was accompanied by a conspicuous reduction in the protein levels of GPX4 and p-AKT in Eca 109 and KYSE 150 cells pathological type in China. Our study substantiates that there is a significant correlation between DNAJB6 expression level and incidence of lymph node metastasis in ESCC patient. This result demonstrates that DNAJB6 may be a biomarker of occult lymph node metastasis (OLM) of ESCC.

Exclusively, our research is the first to give evidence that there is a relationship between DNAJB6 and ferroptosis. Overexpressing DNAJB6a can promote ferroptosis in ESCC by some unknown pathways. Further studies are necessary to explore theirs underlying molecular mechanisms and potential clinical utilities.

\section{Conclusion}

In summary, DNAJB6 is down-regulated in ESCC tissues. Our data suggest that DNAJB6 plays an important anti-oncogenic role in ESCC evolvement via ferroptosis. DNAJB6 may be a potential therapeutic target for the therapy of ESCC.

showed that GPX4 suppresses ferroptosis; in return, inhibiting GPX4 increases lipid peroxidation, which contributes to ferroptotic cancer death [4]. Furthermore, increasing the degradation of GPX4 also contributes to this process [18, 19]. At the same time of ferroptosis, glutathione biosynthesis is inhibited [20]. Besides, some unique changes have taken place in the morphology of mitochondria during the ferroptosis [21], including the loss of structural integrity, such as smaller than normal mitochondria with condensed mitochondrial membrane densities, reduced or absent mitochondria cristae [10, 11], and outer mitochondrial membrane rupture [5].

In order to investigate whether there is a connection between DNAJB6 and ferroptosis, we proceed with relevant experiment. MDA and GSH assays indicated that DNAJB6a overexpression enhanced lipid peroxidation and degraded GSH levels. Western blot results suggested that DNAJB6a overexpression has the ability to down-regulate GPX4. TEM assay also shows mitochondrial morphology as what mentioned before. Therefore, we can conclude that overexpressing DNAJB6a can promote ferroptosis.

Our study proved the DNAJB6 antineoplastic role in ESCC by different methods in vivo and in vitro, based on the research by Yu et al [13], and verified its effect on AKT signaling pathway regulation.

This result is consistent with the study by Mitra A et al [22] that DNAJB6a weakens malignant activity of breast carcinoma. However, DNAJB6 was found to be an oncogene and to aggravate colorectal cancer's invasion according to Zhang et al [23]. In addition, Lin et al [24] found that DNAJB6 can promote cell invasion and migration in HCT 116 cell. This may be due to the tissue-specific character or the gene exist mutation and methylation in diverse tissue.

There are obvious regional characteristics of the incidence of esophagus carcinoma. ESCC is the most common
Funding This work was supported by grants from National Natural Science Foundation of China (81902418), Key R\&D project of Shandong Province (2018GSF118123), Natural Science Foundation of Shandong Province (2014ZRB14125).

\section{Compliance with Ethical Standards}

Conflict of interest The authors declare that they have no conflict of interest.

Ethical approval All procedures performed in studies involving human participants were in accordance with the ethical standards of the Institutional Ethics Committee of Shandong Provincial Hospital Affiliated to Shandong University and with the 1964 Helsinki Declaration and its later amendments or comparable ethical standards. All procedures performed in studies involving animals were in accordance with the ethical standards of Ethics Committee of Shandong Provincial Hospital Affiliated to Shandong University.

Informed consent Additional informed consent was obtained from all individual participants for whom identifying information is included in this article.

Open Access This article is distributed under the terms of the Creative Commons Attribution-NonCommercial 4.0 International License (http://creativecommons.org/licenses/by-nc/4.0/), which permits any noncommercial use, distribution, and reproduction in any medium, provided you give appropriate credit to the original author(s) and the source, provide a link to the Creative Commons license, and indicate if changes were made. 


\section{References}

1. Han-Ze Z, Guang-Fu J, Hong-Bing S. Epidemiologic differences in esophageal cancer between Asian and Western populations. Chin J Cancer. 2012;31:281-286.

2. Winter L, Goldmann WH. Biomechanical characterization of myofibrillar myopathies. Cell Biol Int. 2015;39:361-363.

3. Tarangelo A, Magtanong L, Bieging-Rolett KT, et al. Suppresses metabolic stress-induced ferroptosis in cancer cells. Cell Rep. 2018;22:569-575.

4. Yang W, Sriramaratnam R, Welsch M, et al. Regulation of ferroptotic cancer cell death by GPX4. Cell. 2014;156:317-331.

5. Friedmann Angeli JP, Schneider M, Proneth B, et al. Inactivation of the ferroptosis regulator $\mathrm{Gpx} 4$ triggers acute renal failure in mice. Nat Cell Biol. 2014;76:1180-1191.

6. Ingold I, Berndt $\mathrm{C}$, Schmitt $\mathrm{S}$, et al. Selenium utilization by GPX4 is required to prevent hydroperoxide-induced ferroptosis. Cell. 2017;172:409-422.

7. Rennekamp AJ. The ferrous awakens. Cell. 2017;171:1225.

8. Hangauer MJ, Viswanathan VS, Ryan MJ, et al. Drug-tolerant persister cancer cells are vulnerable to GPX4 inhibition. Nature. 2017;551:247.

9. Dolma S, Lessnick SL, Hahn WC, et al. Identification of genotype-selective antitumor agents using synthetic lethal chemical screening in engineered human tumor cells. Cancer Cell. 2003;3:285-296.

10. Yagoda N, Von Rechenberg M, Zaganjor E, et al. RAS-RAFMEK-dependent oxidative cell death involving voltage-dependent anion channels. NATURE. 2007;447:865-869.

11. Dixon S, Lemberg K, Lamprecht M, et al. Ferroptosis: an iron-dependent form of nonapoptotic cell death. Cell. 2012;149:1060-1072.

12. Martin JC, Herbert BS, Hocevar BA. Disabled-2 downregulation promotes epithelial-to-mesenchymal transition. $\mathrm{Br} J$ Cancer. 2010;103:1716-1723.

13. Yu VZ, Wong VCL, Dai W, et al. Nuclear localization of DNAJB6 is associated with survival of patients with esophageal cancer and reduces AKT signaling and proliferation of cancer cells. Gastroenterology. 2015;149:1825-1836.
14. Mitra A, Menezes ME, Shevde LA, et al. DNAJB6 induces degradation of ?-Catenin and causes partial reversal of mesenchymal phenotype. J Biol Chem. 2010;285:24686-24694.

15. Mitra A, Menezes ME, Pannell LK, et al. DNAJB6 chaperones PP2A mediated dephosphorylation of GSK3 $\beta$ to downregulate $\beta$-catenin transcription target, osteopontin. Oncogene. 2012;31:4472-4483.

16. Yang WS, Stockwell BR. Ferroptosis: death by lipid peroxidation. Trends Cell Biol. 2016;26:165-176.

17. Liang $\mathrm{H}$, Yoo SE, Na R, et al. Short form glutathione peroxidase 4 is the essential isoform required for survival and somatic mitochondrial functions. J Biol Chem. 2009;284:30836-30844.

18. Yu Y, Xie Y, Cao L, et al. The ferroptosis inducer erastin enhances sensitivity of acute myeloid leukemia cells to chemotherapeutic agents. Mol Cell Oncol. 2015;2:e1054549.

19. Shimada K, Skouta R, Kaplan A, et al. Global survey of cell death mechanisms reveals metabolic regulation of ferroptosis. Nat Chem Biol. 2016;12:497.

20. Alvarez SW, Sviderskiy VO, Terzi EM, et al. NFS1 undergoes positive selection in lung tumours and protects cells from ferroptosis. Nature. 2017;551:639.

21. Xie Y, Hou W, Song X, et al. Ferroptosis: process and function. Cell Death Differ. 2016;23:369.

22. Mitra A, Fillmore RA, Metge BJ, et al. Large isoform of MRJ (DNAJB6) reduces malignant activity of breast cancer. Breast Cancer Res BCR. 2008;10:R22.

23. Zhang TT, Jiang YY, Shang L, et al. Overexpression of DNAJB6 promotes colorectal cancer cell invasion through an IQGAP1/ERK-dependent signaling pathway. Mol Carcinog. 2014;54:1205-1213.

24. Lin Y, Peng N, Zhuang H, et al. Heat shock proteins HSP70 and MRJ cooperatively regulate cell adhesion and migration through urokinase receptor. BMC Cancer. 2014;14:639.

Publisher's Note Springer Nature remains neutral with regard to jurisdictional claims in published maps and institutional affiliations. 